Subscriber access provided by Caltech Library

\title{
Letter
}

\section{Bi-containing n-FeWO Thin Films Provide the Largest Photovoltage and Highest Stability for a sub-2 eV Band Gap Photoanode}

Lan Zhou, Aniketa Shinde, Santosh Suram, Helge Stein, Sage Bauers, Andriy Zakutayev, Joseph S DuChene, Guiji Liu, Elizabeth Peterson, Jeffrey B. Neaton, and John M. Gregoire

ACS Energy Lett., Just Accepted Manuscript • DOI: 10.1021/acsenergylett.8b01514 • Publication Date (Web): 12 Oct 2018

Downloaded from http://pubs.acs.org on October 16, 2018

\section{Just Accepted}

"Just Accepted" manuscripts have been peer-reviewed and accepted for publication. They are posted online prior to technical editing, formatting for publication and author proofing. The American Chemical Society provides "Just Accepted" as a service to the research community to expedite the dissemination of scientific material as soon as possible after acceptance. "Just Accepted" manuscripts appear in full in PDF format accompanied by an HTML abstract. "Just Accepted" manuscripts have been fully peer reviewed, but should not be considered the official version of record. They are citable by the Digital Object Identifier (DOI@). "Just Accepted" is an optional service offered to authors. Therefore, the "Just Accepted" Web site may not include all articles that will be published in the journal. After a manuscript is technically edited and formatted, it will be removed from the "Just Accepted" Web site and published as an ASAP article. Note that technical editing may introduce minor changes to the manuscript text and/or graphics which could affect content, and all legal disclaimers and ethical guidelines that apply to the journal pertain. ACS cannot be held responsible for errors or consequences arising from the use of information contained in these "Just Accepted" manuscripts. 


\section{Bi-containing n-FeWO ${ }_{4}$ Thin Films Provide the}

\section{Largest Photovoltage and Highest Stability for a}

\section{sub-2 eV Band Gap Photoanode}

Lan Zhou, ${ }^{a, 1}$ Aniketa Shinde, ${ }^{a, 1}$ Santosh K. Suram, ${ }^{a, 2}$ Helge S. Stein, ${ }^{a}$ Sage R. Bauers, ${ }^{b}$ Andriy

Zakutayev, ${ }^{b}$ Joseph S. DuChene, ${ }^{a, c}$ Guiji Liu, ${ }^{d}$ Elizabeth A. Peterson, ${ }^{d, e}$ Jeffrey B. Neaton, d,e,f

John M. Gregoire ${ }^{a, *}$

a Joint Center for Artificial Photosynthesis, California Institute of Technology; Pasadena,

California 91125, United States; ${ }^{b}$ Materials Science Center, National Renewable Energy

Laboratory, Golden, Colorado 80401, United States; ${ }^{\mathrm{c}}$ Thomas J. Watson Laboratory of Applied

Physics, California Institute of Technology; Pasadena, California 91125, United States; ${ }^{\text {d Joint }}$

Center for Artificial Photosynthesis, Lawrence Berkeley National Laboratory, Berkeley, CA

94720, United States; ${ }^{e}$ Department of Physics, University of California, Berkeley, Berkeley, CA

94720, United States; ${ }^{\mathrm{f}}$ Molecular Foundry, Lawrence Berkeley National Laboratory, Berkeley,

CA 94720, United States; Kavli Energy NanoSciences Institute, University of California,

Berkeley, Berkeley, CA 94720, United States.

\section{Corresponding Author}

*gregoire@caltech.edu

${ }^{1}$ Equal contribution. ${ }^{2}$ current address: Toyota Research Institute, Los Altos, CA 94022, United States. 


\begin{abstract}
Photoelectrocatalysis of the oxygen evolution reaction remains a primary challenge for development of tandem-absorber solar fuels generators due to the lack of a photoanode with broad solar spectrum utilization, large photovoltage, and stable operation. Bismuth vanadate with 2.4$2.5 \mathrm{eV}$ band gap has shown the most promise because its photoactivity down to $0.4 \mathrm{~V}$ vs RHE is sufficiently low to couple to a lower-gap photocathode for fuel synthesis. Through development of photoanodes based on the $\mathrm{FeWO}_{4}$ structure, in particular Fe-rich variants with addition of about $6 \% \mathrm{Bi}$, we demonstrate the same $0.4 \mathrm{~V}$ vs RHE turn-on voltage with a $2 \mathrm{eV}$ band gap metal oxide, enabling a 2-fold increase in the device efficiency limit. Combinatorial exploration of materials composition and processing facilitated synthesis of n-type variants of this typically p-type semiconductor that exhibit much higher photoactivity than previous implementations of $\mathrm{FeWO}_{4}$ in solar photochemistry. The photoanodes are particularly promising for solar fuels applications given their stable operation in acid and base.
\end{abstract}

\title{
TOC GRAPHICS
}

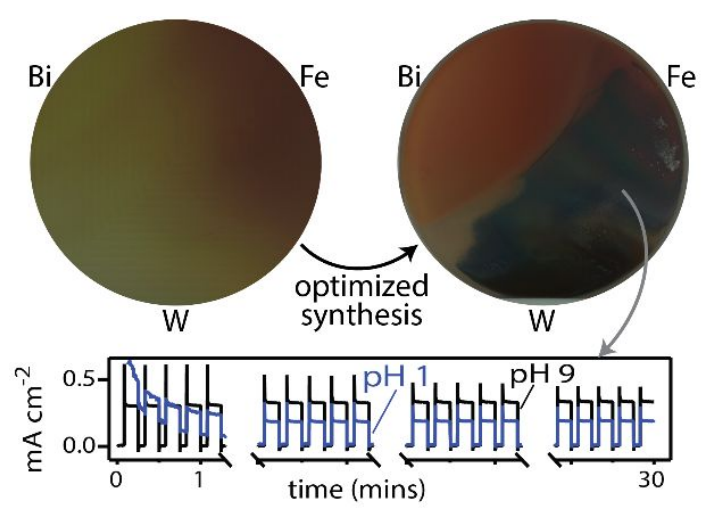


Photoelectrocatalysis of the oxygen evolution reaction (OER) enables harvesting of solar energy for technologies such as solar fuel generation in which water oxidation is coupled to fuel synthesis. ${ }^{1}$ Given their unique oxidative stability, metal oxides are excellent candidate photoanodes but no material to date has met the requirements of (i) effective utilization of the solar spectrum, which requires a band gap energy no larger than about $2 \mathrm{eV}$; (ii) sufficient photovoltage to couple to a photocathode, which for fuel synthesis using a smaller-gap photocathode corresponds to a flat band potential below about $0.6 \mathrm{~V}$ vs RHE; and (iii) stable photoanode operation, which requires either thermodynamic stability or self-passivation. A wide variety of electrodes employing high efficiency semiconductors, such as the recent report of $\mathrm{IrO}_{2}$-protected InGaN, ${ }^{2}$ have demonstrated excellent solar utilization and photovoltage, but the liability of singlepoint defects in the protective layer causing semiconductor corrosion limits the viability of such photoanodes and makes self-passivation a minimum requirement for electrolyte-immersed operation. Many metal oxides meet this requirement but even the few photoactive materials suffer from large band gap energies that lead to ineffective utilization of the solar spectrum and/or insufficient photovoltages for high-efficiency tandem devices. ${ }^{1,3}$

The photoanode requirements noted above are ordered by increasing ability to improve properties through optimization of morphology, doping/alloying, and protective and/or catalytic coatings. For example, alloying and morphology optimization of $\mathrm{BiVO}_{4}$ has improved its initial reported above-gap external quantum efficiency (EQE) from about $10 \%{ }^{4}$ to above $90 \%,{ }^{5}$ and recent optimization of multi-layer coatings has improved stability and lowered the turn-on voltage (lowest potential where photocurrent is observed, which can be no lower than the flat band potential) from $0.4 \mathrm{~V}$ vs RHE to $0.2 \mathrm{~V}$ vs RHE. ${ }^{6}$ However, improving solar spectrum utilization with materials engineering has been relatively unsuccessful, highlighting the importance of 
discovering photoanode materials with low band gap energy that can be further developed into efficient photoanodes. $\mathrm{Fe}_{2} \mathrm{O}_{3}$ and $\mathrm{WO}_{3}$ are classic photoanode materials, making iron tungstates a promising search space for new photoanodes. Previous combinatorial exploration of this space identified modest improvements to photoactivity with mixtures of the binary oxides, ${ }^{7}$ Fe-alloyed $\mathrm{W}_{5} \mathrm{O}_{14},{ }^{8}$ and $\mathrm{Fe}_{2} \mathrm{WO}_{6}{ }^{8}$ However, all reported photoanodes in this composition space suffer from intrinsically limited photovoltage and low carrier diffusion length. ${ }^{9}$ To further explore iron tungstates, we explore their combination with $\mathrm{Bi}$ and employ different processing conditions that prove to be critical for the synthesis of n-type $\mathrm{FeWO}_{4}$, whose $1.95 \pm 0.1 \mathrm{eV}$ band gap, $0.4 \mathrm{~V}$ vs RHE turn-on potential near its $0.35 \pm 0.1 \mathrm{~V}$ vs RHE flat band potential, and self-passivation in both acid and base electrolytes makes it the first metal oxide to meet the 3 primary photoanode requirements.

Exploration of Bi-W-Fe oxides proceeded through synthesis of combinatorial metal oxide libraries using 2 complementary methods to gauge the effects of materials processing. Both libraries were prepared by sputter deposition of metal targets onto a $100 \mathrm{~mm}$-diameter $\mathrm{SnO}_{2}: \mathrm{F}$ (FTO) coated glass substrate and subsequently annealed in air at $610^{\circ} \mathrm{C}$ for 1 hour (see Table S1, ESI). One library was deposited as an oxide using $10 \% \mathrm{O}_{2}$ in the sputter atmosphere, resulting in relatively weak optical absorption (Table S1), whereas the film deposited as a metal using only Ar as sputter gas exhibited high optical absorption over a broad composition range and became the focus of photoelectrochemical (PEC) characterization. Using a previously reported scanning droplet cell for high throughput screening, ${ }^{10}$ characterization of the spectral photoresponse at 1.23 V vs RHE was performed on 215 compositions in aqueous $\mathrm{pH} 9$ borate electrolyte with $0.01 \mathrm{M}$ sodium sulfite as sacrificial hole acceptor (SLF9) using toggled-illumination chronoamperometry (CA). While the photocurrent was measured using 4 light emitting diodes (LEDs) spanning 2.07 to $3.2 \mathrm{eV}$, the photocurrent at $2.4 \mathrm{eV}$ (Figure 1) is particularly important since appreciable 
photoresponse at this photon energy demonstrates utilization of lower-energy photons than stateof-the-art photoanode $\mathrm{BiVO}_{4}$. Such photocurrent was observed near the Fe-W binary line with $\mathrm{Fe}$ concentration between 0.5 and 0.7 and $\mathrm{Bi}$ concentration below 0.2 , prompting the choice of $\mathrm{Fe}_{0.56} \mathrm{~W}_{0.38} \mathrm{Bi}_{0.06} \mathrm{O}_{\mathrm{z}}$ (circled in Figure 1) for further characterization. ${ }^{*}$
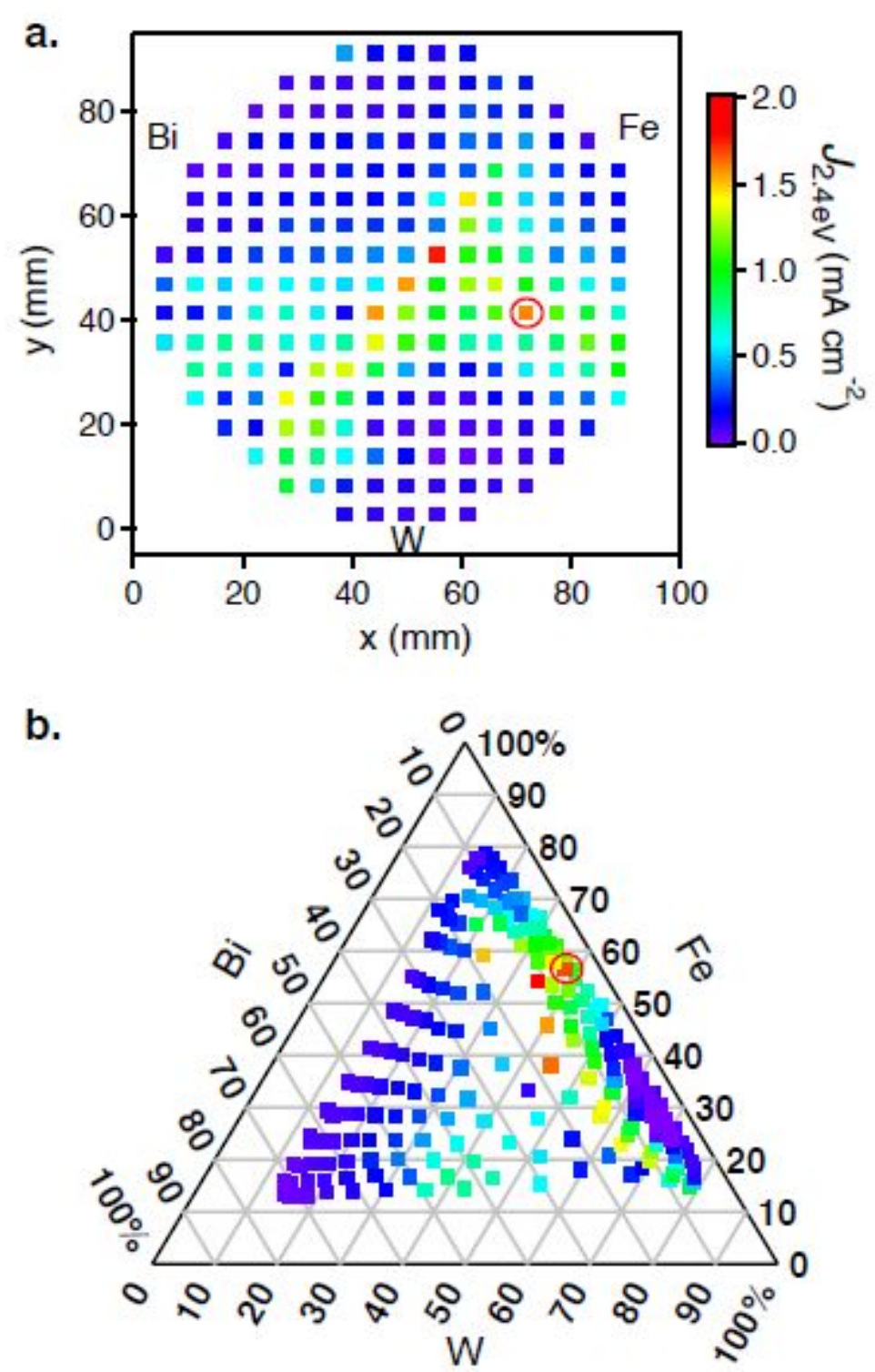

Figure 1. The photocurrent density under $2.4 \mathrm{eV}$ illumination $\left(J_{2.4 \mathrm{eV}}\right)$ mapped over both the library position space (a) and the composition space (b) of the Bi-W-Fe ternary oxide library in aqueous pH 9 borate electrolyte with $0.01 \mathrm{M}$ sodium sulfite. The red circle refers to the $\mathrm{Fe}_{0.56} \mathrm{~W}_{0.38} \mathrm{Bi}_{0.06} \mathrm{O}_{\mathrm{z}}$ 
sample discussed below, and the other high photocurrent density sample $\left(\mathrm{Fe}_{0.54} \mathrm{~W}_{0.35} \mathrm{Bi}_{0.11} \mathrm{O}_{\mathrm{z}}\right)$ is further characterized in Figure S2.

X-ray diffraction (XRD, Figure S4a) characterization of the sample revealed that $\mathrm{FeWO}_{4}$ is the primary phase coexisting with a $\mathrm{Bi}_{2} \mathrm{O}_{3}$ minority phase. The spectral transmittance, total reflectance, and absorption coefficient are shown in Figure $2 \mathrm{a}$ and are assumed to be representative of the $\mathrm{FeWO}_{4}$ structure given the small amount and high transparency of $\mathrm{Bi}_{2} \mathrm{O}_{3}$. The sample exhibits absorption over the entire measured wavelength with absorptivity exceeding $4 \times 10^{4} \mathrm{~cm}^{-1}$ for photon energies above the $1.95 \pm 0.1 \mathrm{eV}$ direct-allowed band gap, determined by Tauc analysis (Figure 2b). Similar band gap energies for $\mathrm{FeWO}_{4}$ have been reported with nanocrystal samples, ${ }^{11}$ but larger band gap energies up to $2.7 \mathrm{eV}$ have also been reported. ${ }^{12-14}$ The external quantum efficiency (EQE, also referred to as the incident photon to current efficiency) from the CAs at 1.23 $\mathrm{V}$ vs RHE is plotted in Figure 2b, revealing photoactivity with the $2.07 \mathrm{eV}$ LED, just above the band gap energy, and higher EQE from 3\% to 6\% at higher photon energies. Direct comparison to other $\sim 2 \mathrm{eV}$ band gap metal oxides is enabled by our previous identification of several copper vanadate photoanodes, ${ }^{3,15}$ and Figure $\mathrm{S} 3$ demonstrates that $\mathrm{Fe}_{0.56} \mathrm{~W}_{0.38} \mathrm{Bi}_{0.06} \mathrm{O}_{z}$ provides a 10-fold improvement in EQE with $2.4 \mathrm{eV}$ illumination. Improved EQE at this photon energy is important not only for defining a path to move beyond $\mathrm{BiVO}_{4}$, but also for differentiating $\mathrm{FeWO}_{4}$ from lowgap oxides like the copper vanadates whose indirect band gap leads to low EQE at photon energies below the considerably higher direct band gap. ${ }^{16}$ 


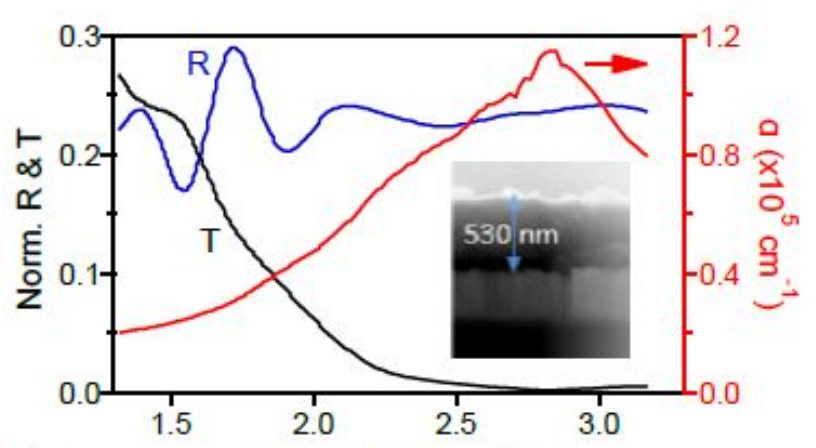

a.

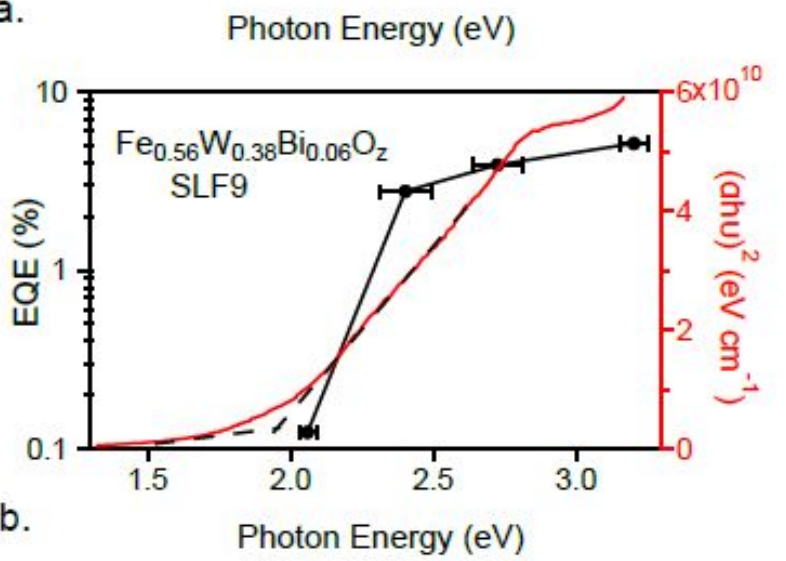

Figure 2. (a) Spectra of the normalized transmittance $\mathrm{T}$, normalized total reflectance R, and absorption coefficient $\alpha$ for $\mathrm{Fe}_{0.56} \mathrm{~W}_{0.38} \mathrm{Bi}_{0.06} \mathrm{O}_{\mathrm{z}}$, calculated using the thickness indicated by the inset cross-section scanning electron microscopy image. (b) Spectral EQE of this sample in sulfitecontaining pH 9 electrolyte using 4 different LEDs $(2.07,2.4,2.74$, and $3.2 \mathrm{eV})$ with horizontal error bars indicating the LED line widths. The direct-allowed Tauc plot (red) indicates a $1.95 \pm 0.1$ $\mathrm{eV}$ direct band gap (intersection of black dashed lines).

With this demonstration of excellent solar spectrum utilization, we turn to characterization of the photovoltage via toggled-illumination cyclic voltammetry $(\mathrm{CV})$ on the $\mathrm{Fe}_{0.56} \mathrm{~W}_{0.38} \mathrm{Bi}_{0.06} \mathrm{O}_{\mathrm{z}}$ sample with $2.4 \mathrm{eV}$ illumination (Figure 3a), both with and without sodium sulfite. The absence of transient photocurrent density spikes under toggled illumination indicates low carrier surface 
recombination, ${ }^{17}$ and the substantially larger photocurrent with the hole scavenger indicates that an OER co-catalyst will be required for deployment as a photoanode. To validate this result from high throughput screening, conventional PEC characterization was performed with front-side AM $1.5 \mathrm{G}$ illumination in the same $\mathrm{pH} 9$ electrolyte both with and without the hole scavenger using a $0.435 \mathrm{~cm}^{2}$ epoxy-encapsulated electrode cut from the library plate. The resulting CVs of Figure $3 \mathrm{~b}$ are in excellent agreement with the corresponding high throughput measurements of Figure $3 \mathrm{a}$. The CVs in SLF9 electrolyte provide the best quantification of the photovoltage provided by this semiconductor, indicating a turn-on potential of approximately $0.4 \mathrm{~V}$ vs RHE, matching the value for optimized, uncoated $\mathrm{BiVO}_{4} \cdot{ }^{6}$ Achieving the same turn-on voltage with $2 \mathrm{eV}$ instead of 2.4-2.5 $\mathrm{eV}$ band gap has significant implications for tandem device efficiency. Using the Fountaine et al. ${ }^{18}$ model for tandem photoelectrochemical water splitting cells, this corresponds to a more than doubling of the maximum device efficiency, from approximately $8 \%$ to $18 \%$ under "ideal" conditions or approximately $7 \%$ to $16 \%$ under "realistic" conditions.

With this demonstrated ability to enable high efficiency tandem solar fuels generators, we investigate the stability criterion, starting with the calculated Materials Project Pourbaix energetics of the $\mathrm{FeWO}_{4}$ system in Figure S9. ${ }^{19} \mathrm{Like} \mathrm{BiVO}_{4}, \mathrm{FeWO}_{4}$ is only thermodynamically stable at moderate $\mathrm{pH}$ and low potential, requiring passivation or protection layers to achieve stable photoanode operation. ${ }^{20} \mathrm{~A}$ desirable quality of the $\mathrm{FeWO}_{4}$ Pourbaix diagram is the availability of anodic protection layers that may provide self-passivation. At OER potentials, $\mathrm{Fe}_{2} \mathrm{O}_{3}$ is a stable self-passivation layer above $\mathrm{pH} 1$, and $\mathrm{WO}_{3}$ is a stable self-passivation layer below $\mathrm{pH}$. From this analysis, the $\mathrm{FeWO}_{4}$-based photoanode should also be operable in strong acid, which we evaluated using a neighboring sample with as-synthesized composition $\mathrm{Fe}_{0.59} \mathrm{~W}_{0.35} \mathrm{Bi}_{0.06} \mathrm{O}_{\mathrm{z}}$ by performing a toggled illumination $\mathrm{CV}$ in $0.1 \mathrm{M} \mathrm{H}_{2} \mathrm{SO}_{4}$ with $0.1 \mathrm{M}$ methanol added as a hole 
scavenger (MET1.4, Figure S1a). While the photocurrent density is lower in the pH 1.4 electrolyte, stable photocurrent during $30 \mathrm{~min}$ toggled-illumination at $1.23 \mathrm{~V}$ vs RHE indicates successful passivation in strong acid (Figure S1b).
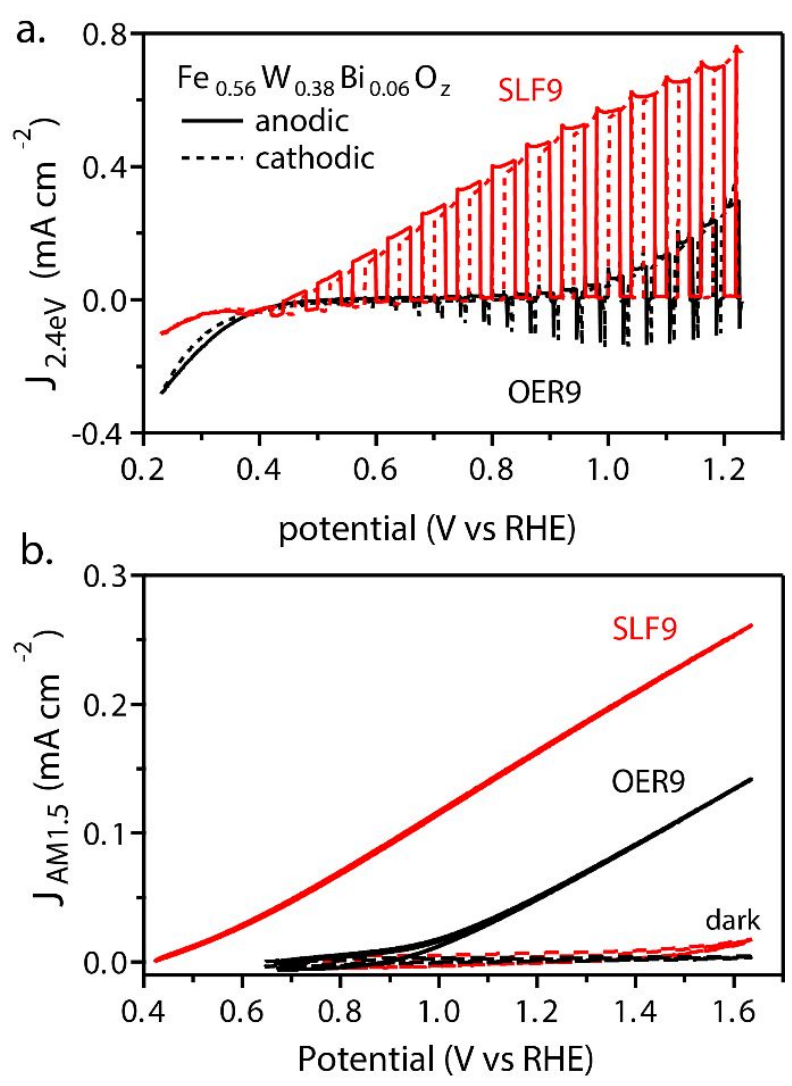

Figure 3. (a) Front-side, toggled-illumination (2.4 eV LED) CVs of sample $\mathrm{Fe}_{0.56} \mathrm{~W}_{0.38} \mathrm{Bi}_{0.06} \mathrm{O}_{z}$ starting with a cathodic sweep from $1.23 \mathrm{eV}$ vs RHE (dashed lines) followed by the return anodic sweep (solid lines) in aqueous pH 9 borate electrolyte with (SLF9, red) and without (OER9, black) sodium sulfite. (b) $\mathrm{CV}$ cycles of the epoxy-encapsulated electrode (area $0.435 \mathrm{~cm}^{2}$ ) containing the $\mathrm{Fe}_{0.56} \mathrm{~W}_{0.38} \mathrm{Bi}_{0.06} \mathrm{O}_{z}$ sample in SLF9 electrolyte and subsequently in OER9 electrolyte. In each electrolyte, first 2 cycles were acquired with no illumination ("dark", dashed lines) and subsequent 2 cycles were collected under AM $1.5 \mathrm{G}, 100 \mathrm{~mW} \mathrm{~cm}^{-2}$ front-side illumination with a scan rate of $0.05 \mathrm{~V} \mathrm{~s}^{-1}$. 
To assess longer-term stability we use SLF9, the electrolyte that provided the highest photocurrent. Using a $\mathrm{Fe}_{0.59} \mathrm{~W}_{0.36} \mathrm{Bi}_{0.05} \mathrm{O}_{\mathrm{z}}$ electrode, the following electrochemical procedure was repeated 34 times over the course of 7 days of testing (10 calendar days): a CV similar to that of Figure 3a was followed by approximately $50 \mathrm{~min}$ CA at $1.23 \mathrm{~V}$ vs RHE during which the sample was illuminated $97 \%$ of the time with a broadband light source (W35, Doric LEDc2, $107 \mathrm{~mW} \mathrm{~cm}^{-2}$ irradiance). Combined with XRF and SEM characterization, the results of Figure 4 indicate no detectable corrosion or changes in film morphology during extensive photoelectrochemical operation. The evolution of the photoactivity is somewhat surprising, with loss of approximately $20 \%$ of photocurrent through the course of the day with recovery upon exposure to air overnight. Inspection of toggled illumination CVs (Figure 4b) reveals that the degradation of photoactivity during the day is marked by a substantial change in CV shape including increased transients upon illumination toggling, which suggests a modification to the surface that introduces surface states and increases carrier recombination. Additionally considering that X-ray photoelectron spectroscopy (XPS, Figure S5, ESI) of the $\mathrm{Fe}_{0.56} \mathrm{~W}_{0.38} \mathrm{Bi}_{0.06} \mathrm{O}_{z}$ sample PEC operation revealed a predominantly $\mathrm{Fe}^{+3}$ surface with $\mathrm{Bi}$ and $\mathrm{W}$ concentrations on the order of 1 at.\%, and that $\mathrm{Fe}_{2} \mathrm{O}_{3}$ was identified as an impurity phase, these results are consistent with the formation of near-surface $\mathrm{FeOOH}^{21}$ during PEC operation that restores to $\mathrm{Fe}_{2} \mathrm{O}_{3}$ in air, with characteristic time scales that are larger than 1 hour. In this case, the $\mathrm{FeOOH}$ is deleterious to PEC performance and should be mitigated in future design of surface coatings. 

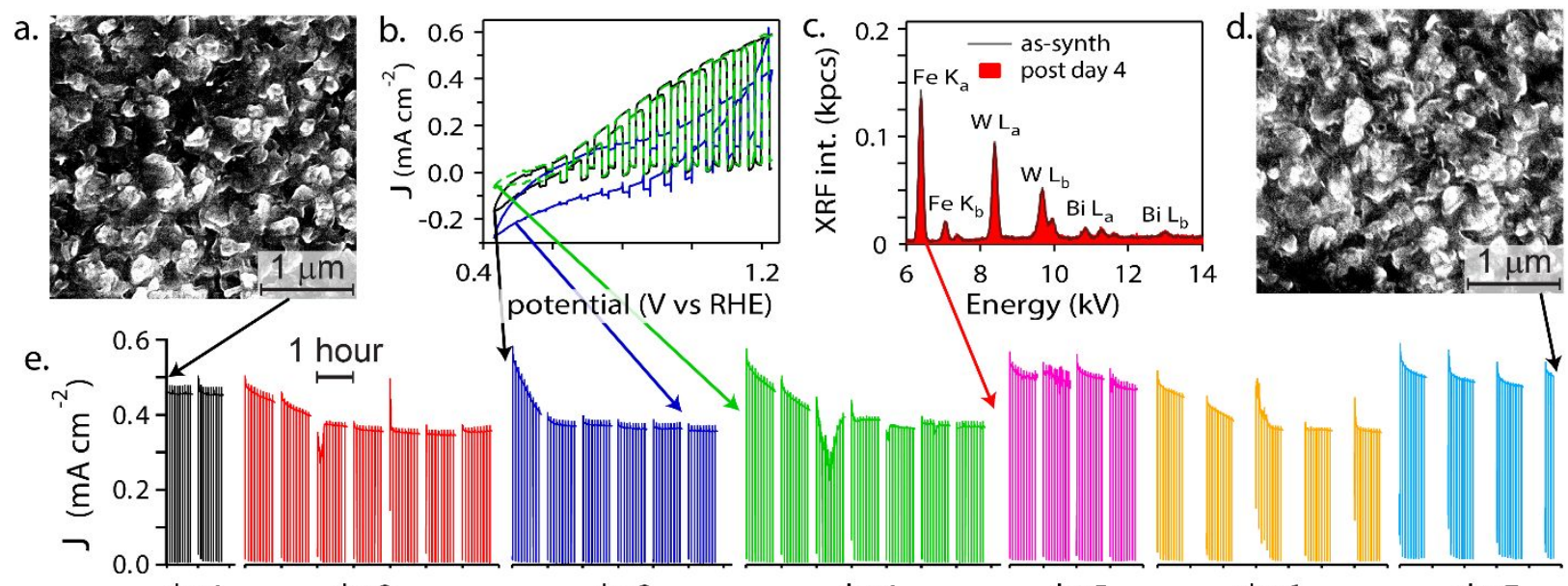

Figure 4. Stability measurement of $\mathrm{Fe}_{0.59} \mathrm{~W}_{0.36} \mathrm{Bi}_{0.05} \mathrm{O}_{z}$ electrode in SLF9 electrolyte over the course of 7 days with additional characterizations performed at time points indicated by the respective arrows. (a) SEM image of a nearby sample that was not electrochemically tested and thus representative of the electrode at the start of electrochemical testing. (b) 3 representative toggled-illumination CVs recorded at the beginning of day 3 (black), after 5 hours of testing on day 3 (blue), and at the beginning of day 4 (green). (c) XRF spectrum taken at the end of day 4 and comparison to the spectrum of the as-synthesized spectrum. (d) SEM image of the electrode after completion of electrochemical testing, and (e) the current density under toggled-illumination white light (97\% on, $3 \%$ off) at $1.23 \mathrm{~V}$ vs RHE. Air exposure, in particular the 70 hours between days 4 and 5, improves initial photocurrent and slows photocurrent degradation for subsequent experiments (see SI).

The XPS-identification of an Fe-enriched surface additionally brings into question the role of surface-segregated layers during synthesis. Importantly, the $\mathrm{FeWO}_{4}$ phase was not observed at any cation composition in the library deposited with $\mathrm{O}_{2}$ present in the sputter atmosphere, indicating that for annealing in air, this phase is only accessible when starting with an oxygen-depleted thin 
film. Indeed, inspection of the solid state thermodynamics (Figure S10) reveals that at $610{ }^{\circ} \mathrm{C}$, $\mathrm{FeWO}_{4}$ is the equilibrium phase for $\mathrm{O}_{2}$ pressure below $1 \times 10^{-4} \mathrm{~atm}$, well below the $\sim 2 \times 10^{-1}$ atm of the air annealing in this work. The film deposited with oxygen is in better agreement with the equilibrium phase behavior after annealing, with XRD characterization revealing formation of $\mathrm{Fe}^{+3}$ containing phases such as $\mathrm{Fe}_{2} \mathrm{~W}_{3} \mathrm{O}_{12}$ and $\mathrm{Fe}_{2} \mathrm{WO}_{6}$. Starting with metal thin films enables access to the $\mathrm{FeWO}_{4}$ phase that is far from equilibrium in the air anneal, suggesting the presence of a surface layer that limits oxygen transport to the underlying film during annealing. To explore the presence of such a film, a Fe-W library (omitting Bi) was synthesized by performing the same $610{ }^{\circ} \mathrm{C}$ air anneal of a metal composition spread (see Table S1). Similar highly-absorbing material was observed for the films with thickness above approximately $500 \mathrm{~nm}$, with XRD analysis revealing that $\mathrm{FeWO}_{4}$ was only obtained as a majority phase in this thicker portion of the library, further suggesting the importance of limiting oxygen diffusion since thinner films are more amenable to equilibration with the annealing atmosphere. The highest phase concentration of $\mathrm{FeWO}_{4}$ was observed at $\mathrm{Fe}_{0.62} \mathrm{~W}_{0.38} \mathrm{O}_{\mathrm{z}}$, whose $\mathrm{XRD}$ characterization is shown in Figure $\mathrm{S} 4 \mathrm{~b}$ and reveals a presence of approximately $4 \% \mathrm{Fe}_{2} \mathrm{O}_{3}$. PEC characterization (Figure 5a) of this film demonstrated stable photoanodic performance in 4 different electrolytes: $\mathrm{pH} 1.4$ and $\mathrm{pH} 6.6$ containing a hole scavenger and $\mathrm{pH} 3$ and $\mathrm{pH} 9$ without a hole scavenger. Additional PEC characterization is provided in Figure S7 and demonstrates that while photocurrent is lower than with the Bicontaining film, the photo-response of these films is due to $\mathrm{FeWO}_{4}$ as similar performance is obtained for its phase mixture with either $\mathrm{Fe}_{2} \mathrm{O}_{3}$ or $\mathrm{WO}_{3}$. Given the higher photocurrent with the Fe-W-Bi sample, the role of $\mathrm{Bi}$ may be related to passivation of surface defects and/or enhancement of carrier transport, motivating future investigation of the precise location of $\mathrm{Bi}$ in the heterogeneous film. 
In total, we have observed photoactivity of electrodes with $\mathrm{FeWO}_{4}$ majority phase and $\mathrm{Bi}_{2} \mathrm{O}_{3}$, $\mathrm{Fe}_{2} \mathrm{O}_{3}$, or $\mathrm{WO}_{3}$ minority phases. The most likely common role of these 3 different minority phases is surface segregation of a high-valent oxide during synthesis that limits oxygen diffusion and enables growth of $\mathrm{FeWO}_{4}$, which is supported by XPS depth profiling on the as-annealed $\mathrm{Fe}_{0.62} \mathrm{~W}_{0.38} \mathrm{O}_{z}$ film (Figure $\mathrm{S} 6$ ). The near-surface layer contains primarily $\mathrm{Fe}^{+3}$, and film etching leads to increased $\mathrm{W}$ signal and shift of Fe peaks to lower-valent values. This non-equilibrium synthesis also appears to be critical for attaining the n-type conductivity inherent to the photoanodic response of the films. Studies of single and polycrystalline samples identified FeWO as a p-type semiconductor, ${ }^{22,23}$ and Hoang et al. ${ }^{24}$ identified the origins of the p-type conductivity through calculation of the formation energy of point defects and small polarons. As described in detail in Figure S14, at typical $\mathrm{FeWO}_{4}$ synthesis conditions, Fe vacancies (p-type dopants) are the lowest energy point defects and hole polarons have lower formation than electron polarons. At higher Fe chemical potentials corresponding to our synthesis, oxygen vacancies (n-type dopants) are the point defects with lowest formation energy. Given the limited diffusion of oxygen into the film during annealing, oxygen vacancies are the most likely source of n-type doping, and the calculations indicate that electron polarons play an important role in n-type conductivity, especially considering their low trapping energy $(0.17 \mathrm{eV})$ and migration barrier $(0.12 \mathrm{eV})$, which are each lower than that of hole polarons in $\mathrm{FeWO}_{4}{ }^{24}$ While $\mathrm{FeWO}_{4}$ has been used previously for photochemistry, the type of doping in these materials is not always clear as $\mathrm{FeWO}_{4}$ is typically reported to inject holes via its heterojunction with an n-type semiconductor such as $\mathrm{TiO}_{2},{ }^{11} \mathrm{ZnO},{ }^{12}$ and $\mathrm{Co}_{2} \mathrm{O}_{3} \cdot{ }^{25}$ Heterojunctions with $\mathrm{Fe}_{3} \mathrm{O}_{4}$ have resulted in both n-type ${ }^{13}$ and $\mathrm{p}$-type ${ }^{26}$ behavior. The only strong indications of n-type $\mathrm{FeWO}_{4}$ in previous reports has been via nanoparticle synthesis in which the origins of n-type doping are not discussed. ${ }^{11,12,25}$ 
Hoang et al. ${ }^{24}$ also investigated the electronic structure of ferromagnetic (FM) $\mathrm{FeWO}_{4}$, and we performed complementary density functional theory (DFT) calculations with antiferromagnetic (AFM) ordering to assess any difference in the electronic structure since the material is paramagnetic at room temperature. ${ }^{27}$ Our DFT calculations (Figure S11) reveal highly localized Fe $3 d$ states near and at the VBM and strong $\mathrm{W} d$ character near the CBM with the only substantial influence of magnetic ordering being the expected change in the spin channel of the Fe $3 \mathrm{~d}$ states. Similar flat Fe-based valence bands are observed in both FM and AFM band diagrams, suggesting low mobility for band transport and further supporting the importance of both hole and electron polarons in charge transport. Optimization of photocurrent will require materials engineering based on further understanding of polaron conduction in $\mathrm{FeWO}_{4}$.

Given that our photoanodes comprise the first demonstration of n-FeWO 4 thin films, we continue characterization of this doping through a series of experiments starting with Mott-Schottky analysis of a $\mathrm{Fe}_{0.62} \mathrm{~W}_{0.38} \mathrm{O}_{\mathrm{z}}$ film similar to that analyzed by XPS and PEC (Figures S6-S7). The Mott-Schottky plot in Figure 5b confirms the n-type conductivity of the photoelectrode. The data, as well as analogous data acquired at different frequencies (Figure S12), suggest a flat band potential of $0.35 \pm 0.1 \mathrm{~V}$ vs RHE, which is in excellent agreement with the turn-on voltage in Figure 3 but is not conclusive given that this film, like each of our n-FeWO films, contains an impurity phase. To establish whether a more traditional synthesis of the $\mathrm{FeWO}_{4}$ phase would yield p-type conductivity as expected, a film was deposited in $1 \% \mathrm{O}_{2}$ and annealed at $610{ }^{\circ} \mathrm{C}$ in an inert $\mathrm{Ar}$ atmosphere (see Table S1), yielding a phase-pure $\mathrm{FeWO}_{4}$ whose Mott-Schottky analysis reveals p-type conductivity with flat band potential of $2.1 \pm 0.1 \mathrm{~V}$ vs RHE (Figure $5 \mathrm{~b}$ ). The $\sim 1.7 \mathrm{~V}$ difference between the flat band potentials indicates that both $\mathrm{n}-\mathrm{FeWO}_{4}$ and $\mathrm{p}-\mathrm{FeWO}_{4}$ have their Fermi energy within $0.3 \mathrm{~V}$ of the respective band edge, and while $\mathrm{FeWO}_{4}$ has a deep valence band, 

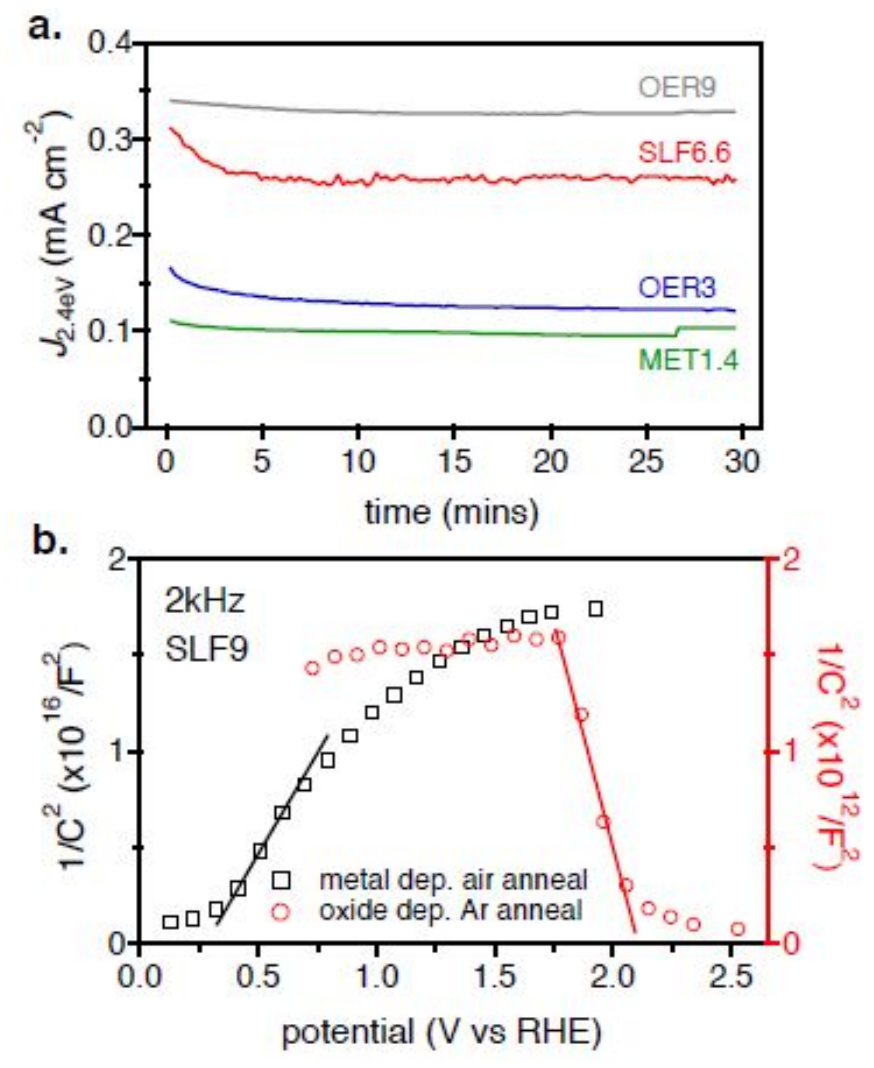

the high flat band energy in the n-FeWO $\mathrm{W}_{4}$ film enables the large photovoltage observed in PEC measurements.

Figure 5. (a) 30 mins PEC stability measurements at applied potential of $1.23 \mathrm{~V}$ vs RHE in various aqueous electrolytes. (b) Mott-Schottky plots for two $\mathrm{FeWO}_{4}$ electrodes with different synthesis methods acquired in SLF9 electrolyte at $2 \mathrm{kHz}$.

To provide additional characterization of the $\mathrm{n}-\mathrm{FeWO}_{4}$, films were prepared on insulating substrates so that electrical characterization could proceed without interference of the FTO conducting back contact. Using a Si wafer with thermal oxide coating, the Fe-W metal was deposited and annealed at $610{ }^{\circ} \mathrm{C}$ in air, resulting in the fortuitous synthesis of a phase-pure $\mathrm{FeWO}_{4}$ film. It appeared that the Fe-rich coating observed previously formed and subsequently delaminated, perhaps during film cooling, such that the remaining film experienced similar non- 
equilibrium synthesis conditions described above but with no detectable $\mathrm{Fe}_{2} \mathrm{O}_{3}$ in $\mathrm{XRD}$ measurements due to the partial delamination. While the doping level may vary from the photoanode films due to this difference in synthesis, the measured Seebeck coefficient of $-77.1 \pm$ $0.5 \mu \mathrm{V} \mathrm{K}^{-1}$ unambiguously demonstrates the n-type doping of the $\mathrm{FeWO}_{4}$ in the absence of $\mathrm{Fe}_{2} \mathrm{O}_{3}$ (Figure S8).

The opportunities for future photoelectrode development are (i) tailored surface coatings to manage surface recombination and increase catalytic activity and (ii) optimize electrode composition and morphology to enhance polaron-based conductivity, all while maintaining n$\mathrm{FeWO}_{4}$ as the primary absorber material. This future work is well motivated by seminal discovery of a low-band gap, high-photovoltage, and stable metal oxide photoanode that establishes $\mathrm{FeWO}_{4}-$ based photoanodes as the most promising route to efficient fuel generation employing solar photochemistry.

\section{ASSOCIATED CONTENT}

\section{Supporting Information.}

The Supporting Information is available free of charge on the ACS Publications website at DOI:XXXX.

Experimental and computational details, additional photoelectrochemical and materials characterization data.

\section{AUTHOR INFORMATION}

\section{Corresponding Author}


*gregoire@caltech.edu

\section{ORCID}

Lan Zhou: 0000-0002-7052-266X

Aniketa Shinde: 0000-0003-2386-3848

Santosh K. Suram: 0000-0001-8170-2685

Helge S. Stein: 0000-0002-3461-0232

Sage R. Bauers:

Andriy Zakutayev: 0000-0002-3054-5525

Joseph S. Duchene:

Guiji Liu:

Elizabeth A. Peterson:

Jeffrey B. Neaton: 0000-0001-7585-6135

John M. Gregoire: 0000-0002-2863-5265

\section{Author Contributions}

1. Lan Zhou and Aniketa Shinde contributed equally.

\section{Notes}

The authors declare no competing financial interest.

The oxygen stoichiometry was not measured. XRD and XPS characterization of average cation valences indicate that $z$ is approximately 2 for all compositions discussed in the manuscript.

\section{ACKNOWLEDGMENTS}

This material is based upon work performed by the Joint Center for Artificial Photosynthesis, a DOE Energy Innovation Hub, supported through the Office of Science of the U.S. Department of Energy under Award Number DE-SC0004993. Computational work at the Molecular Foundry was supported by the Office of Science, Office of Basic Energy Sciences, of the US DOE under 
Contract DE-AC02-05CH11231. Seebeck and resistivity characterization were supported by the U.S. Department of Energy under Contract No. DE-AC36-08GO28308 with Alliance for Sustainable Energy, LLC, the Manager and Operator of the National Renewable Energy Laboratory; funding provided by Office of Science, Office of Basic Energy Sciences, as part of the Energy Frontier Research Center "Center for Next Generation of Materials Design: Incorporating Metastability". We thank Dr. Mitsutaro Umehara for assistance with collection of cross-section SEM images.

\section{REFERENCES}

(1) Sivula, K.; van de Krol, R. Semiconducting Materials For Photoelectrochemical Energy Conversion. Nat. Rev. Mater. 2016, 1 (2), 15010.

(2) Chu, S.; Vanka, S.; Wang, Y. C.; Gim, J.; Wang, Y. J.; Ra, Y. H.; Hovden, R.; Guo, H.; Shih, I.; Mi, Z. T. Solar Water Oxidation by an InGaN Nanowire Photoanode with a Bandgap of 1.7 eV. ACS Energy Lett. 2018, 3 (2), 307-314.

(3) Yan, Q. M.; Yu, J.; Suram, S. K.; Zhou, L.; Shinde, A.; Newhouse, P. F.; Chen, W.; Li, G.; Persson, K. A.; Gregoire, J. M.; et al. Solar Fuels Photoanode Materials Discovery by Integrating High-throughput Theory and Experiment. Proc. Natl. Acad. Sci. U.S.A. 2017, 114 (12), 30403043.

(4) Sayama, K.; Nomura, A.; Zou, Z. G.; Abe, R.; Abe, Y.; Arakawa, H. Photoelectrochemical Decomposition of Water on Nanocrystalline $\mathrm{BiVO}_{4}$ Film Electrodes Under Visible Light. Chem. Commun. 2003, (23), 2908-2909.

(5) Tolod, K. R.; Hernandez, S.; Russo, N. Recent Advances in the $\mathrm{BiVO}_{4}$ Photocatalyst for SunDriven Water Oxidation: Top-Performing Photoanodes and Scale-Up Challenges. Catalysts 2017, 7 (1), 13.

(6) Kim, T. W.; Ping, Y.; Galli, G. A.; Choi, K. S. Simultaneous Enhancements in Photon Absorption and Charge Transport of Bismuth Vanadate Photoanodes For Solar Water Splitting. Nat. Commun. 2015, 6, 8769.

(7) Kollender, J. P.; Mardare, A. I.; Hassel, A. W. Localized Photoelectrochemistry on a Tungsten Oxide-Iron Oxide Thin Film Material Library. ACS Comb. Sci. 2013, 15 (12), 601-608. (8) Meyer, R.; Sliozberg, K.; Khare, C.; Schuhmann, W.; Ludwig, A. High-Throughput Screening of Thin-Film Semiconductor Material Libraries II: Characterization of Fe-W-O Libraries. ChemSusChem 2015, 8 (7), 1279-1285.

(9) Abdi, F. F.; Chemseddine, A.; Berglund, S. P.; van de Krol, R. Assessing the Suitability of Iron Tungstate $\left(\mathrm{Fe}_{2} \mathrm{WO}_{6}\right)$ as a Photoelectrode Material for Water Oxidation. J. Phys. Chem. C 2017, 121 (1), 153-160. 
(10) Gregoire, J. M.; Xiang, C.; Liu, X.; Marcin, M.; Jin, J. Scanning Droplet Cell for High Throughput Electrochemical and Photoelectrochemical Measurements. Rev. Sci. Instrum. 2013, 84 (2), 024102.

(11) Bera, S.; Rawal, S. B.; Kim, H. J.; Lee, W. I. Novel Coupled Structures of $\mathrm{FeWO}_{4} / \mathrm{TiO}_{2}$ and $\mathrm{FeWO}_{4} / \mathrm{TiO}_{2} / \mathrm{CdS}$ Designed for Highly Efficient Visible-Light Photocatalysis. ACS Appl. Mater. Interfaces 2014, 6 (12), 9654-9663.

(12) Ma, Y. C.; Guo, Y. H.; Jiang, H. Y.; Qu, D.; Liu, J.; Kang, W. K.; Yi, Y.; Zhang, W.; Shi, J. S.; Han, Z. Z. Preparation of Network-Like ZnO-FeWO 4 Mesoporous Heterojunctions With Tunable Band Gaps and Their Enhanced Visible Light Photocatalytic Performance. New J. Chem. 2015, 39 (7), 5612-5620.

(13) Sadiq, M. M. J.; Shenoy, U. S.; Bhat, D. K. Enhanced Photocatalytic Performance of NDoped Rgo-FeWO $\mathrm{W}_{4} / \mathrm{Fe}_{3} \mathrm{O}_{4}$ Ternary Nanocomposite in Environmental Applications. Mater. Today Chem. 2017, 4, 133-141.

(14) Wang, H. X.; Wang, C. H.; Cui, X. M.; Qin, L.; Ding, R. M.; Wang, L. C.; Liu, Z.; Zheng, Z. F.; Lv, B. L. Design and Facile One-Step Synthesis of FeWO $\mathrm{W}_{4} / \mathrm{Fe}_{2} \mathrm{O}_{3}$ Di-Modified $\mathrm{WO}_{3}$ With Super High Photocatalytic Activity Toward Degradation of Quasi-Phenothiazine Dyes. Appl. Catal., B 2018, 221, 169-178.

(15) Zhou, L.; Yan, Q. M.; Shinde, A.; Guevarra, D.; Newhouse, P. F.; Becerra-Stasiewicz, N.; Chatman, S. M.; Haber, J. A.; Neaton, J. B.; Gregoire, J. M. High Throughput Discovery of Solar Fuels Photoanodes in the $\mathrm{CuO}-\mathrm{V}_{2} \mathrm{O}_{5}$ System. Adv. Energy Mater. 2015, 5 (22), 1500968.

(16) Jiang, C. M.; Farmand, M.; Wu, C. H.; Liu, Y. S.; Guo, J. H.; Drisdell, W. S.; Cooper, J. K.; Sharp, I. D. Electronic Structure, Optoelectronic Properties, and Photoelectrochemical Characteristics of $\gamma-\mathrm{Cu}_{3} \mathrm{~V}_{2} \mathrm{O}_{8}$ Thin Films. Chem. Mater. 2017, 29 (7), 3334-3345.

(17) Klahr, B.; Gimenez, S.; Fabregat-Santiago, F.; Bisquert, J.; Hamann, T. W. Photoelectrochemical and Impedance Spectroscopic Investigation of Water Oxidation with "CoPi"-Coated Hematite Electrodes. J. Am. Chem. Soc. 2012, 134 (40), 16693-16700.

(18) Fountaine, K. T.; Lewerenz, H. J.; Atwater, H. A. Efficiency Limits for Photoelectrochemical Water-Splitting. Nat. Commun. 2016, 7, 13706.

(19) Singh, A. K.; Zhou, L.; Shinde, A.; Suram, S. K.; Montoya, J. H.; Winston, D.; Gregoire, J. M.; Persson, K. A. Electrochemical Stability of Metastable Materials. Chem. Mater. 2017, 29 (23), 10159-10167.

(20) Toma, F. M.; Cooper, J. K.; Kunzelmann, V.; McDowell, M. T.; Yu, J.; Larson, D. M.; Borys, N. J.; Abelyan, C.; Beeman, J. W.; Yu, K. M.; et al. Mechanistic Insights into Chemical and Photochemical Transformations of Bismuth Vanadate Photoanodes. Nat. Commun. 2016, 7 , 12012.

(21) Louie, M. W.; Bell, A. T. An Investigation of Thin-Film Ni-Fe Oxide Catalysts for the Electrochemical Evolution of Oxygen. J. Am. Chem. Soc. 2013, 135 (33), 12329-12337. (22) Sieber, K.; Kourtakis, K.; Kershaw, R.; Dwight, K.; Wold, A. Preparation and

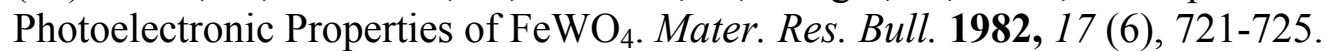
(23) Schmidbauer, E.; Schanz, U.; Yu, F. J. Electrical Transport-Properties of Monocrystalline and Polycrystalline FeWO 4 . J. Phys.: Condens. Matter 1991, 3 (28), 5341-5352.

(24) Hoang, K. Polaron Formation, Native Defects, and Electronic Conduction in Metal Tungstates. Phy. Rev. Mater. 2017, 1 (2), 024603.

(25) Chakraborty, A. K.; Islam, M. R.; Uddin, M. H.; Rhaman, M. M. Novel Visible-LightDriven Photocatalyst $\mathrm{Co}_{3} \mathrm{O}_{4} / \mathrm{FeWO}_{4}$ for Efficient Decomposition of Organic Pollutants. $J$. Cluster Sci. 2018, 29 (1), 67-74. 
(26) Cao, X.; Chen, Y.; Jiao, S. H.; Fang, Z. X.; Xu, M.; Liu, X.; Li, L.; Pang, G. S.; Feng, S. H. Magnetic Photocatalysts With a P-N Junction: $\mathrm{Fe}_{3} \mathrm{O}_{4}$ Nanoparticle and $\mathrm{FeWO}_{4}$ Nanowire Heterostructures. Nanoscale 2014, 6 (21), 12366-12370.

(27) Zhou, Y. X.; Yao, H. B.; Zhang, Q.; Gong, J. Y.; Liu, S. J.; Yu, S. H. Hierarchical FeWO 4 Microcrystals: Solvothermal Synthesis and Their Photocatalytic and Magnetic Properties. Inorg. Chem. 2009, 48 (3), 1082-1090. 